\title{
Expiratory Muscle Strength Training
}

National Cancer Institute

\section{Source}

National Cancer Institute. Expiratory Muscle Strength Training. NCI Thesaurus. Code C125716.

High-intensity, low-repetition expiratory exercises designed to promote strength in the expiratory muscles. Expiratory muscle strength training typically uses a pressure threshold device with a regimented treatment protocol. This treatment may benefit individuals with spinal cord injury, chronic obstructive pulmonary disease, multiple sclerosis, Parkinson's disease, voice disorders, and the sedentary elderly. 